\title{
Batch Transfer of LIGA Microstructures by Selective Electroplating and Bonding
}

\author{
Li-Wei Pan and Liwei Lin
}

\begin{abstract}
A flip-chip, batch transfer process for LIthographie, Galvanoformung and Abformung (LIGA) microstructures has been demonstrated by selective electroplating and bonding. Single layer LIGA microstructures with thickness of $200 \mu \mathrm{m}$ are fabricated on a dummy substrate first. They are then batch transferred to an IC substrate by means of bonding via electroplating. After the selective bonding process, the originally fixed microstructures become free-standing, moveable devices. The electroplating and bonding process is conducted at $50^{\circ} \mathrm{C}$ with applied electroplating current density at $68.3 \mathrm{AM}^{2}$ and it takes about 80 minutes to complete the bonding process. Experimentally, an electrothermally-driven LIGA microgripper has been demonstrated to operate after the batch transfer process. When a maximum input current of $1.6 \mathrm{amp}$ is applied, the tip of the microgripper moves $92 \mu \mathrm{m}$. This flip-chip assembly process enables a new class of integrated electro-mechanical manufacturing at a low processing temperature massively and in parallel. [551]
\end{abstract}

Index Terms-Bonding, electroplating, flip-chip, Lithographie Galvanoformung and Abformung microgripper.

\section{INTRODUCTION}

$\mathbf{P}$ ROCESS integration for micromechanical structures and microelectronics has been one of the most important manufacturing issues in the field of microelectromechanical systems (MEMS). For example, Analog Devices Inc. and Berkeley Sensor and Actuator Center have integrated processes for surface-micromachined polysilicon microstructures with bipolar metal oxide silicon (BIMOS) [1] and CMOS [2], respectively. The integrated single crystal reactive etching and metallization (SCREAM) process has been developed at Cornell University for integrating microelectronics with single crystalline silicon [3]. Sandia National Laboratory has also demonstrated an embedded monolithic integration process for microstructures with CMOS [4]. All of these integrated processes are designed for silicon based microstructures with thickness of less than $20 \mu \mathrm{m}$.

For processes and materials that are not compatible with IC manufacturing, such as Lithographie Galvanoformung and Abformung (LIGA), LIGA-like, and micro electrical discharge machining (EDM) processes, batch transfer of microstructures with substrates that have pre-fabricated microelectronics is a crucial step in building up a complete system. Without the integration process, microstructures fabricated by these

Manuscript received March 20, 2000; revised August 16, 2000. This project is supported in part by a NSF award (DMI-0096220) and a DARPA grant under the MTO/MEMS program. Subject Editor, N. deRooij.

L.-W. Pan is with the Mechanical Engineering and Applied Mechanics, University of Michigan, MI 48109 USA.

L. Lin is with the Mechanical Engineering, University of California, Berkeley, CA 94720 USA (e-mail: lwpan@me.berkeley.edu).

Publisher Item Identifier S 1057-7157(01)01587-6. processes have very limited applications. Previously, efforts in the area of batch assembly have been reported to address this problem, such as solder bump bonding [5], conductive polymer bumps [6], and diffusion bonding [7]. These processes aim to provide reliable electro-mechanical integration. However, drawbacks can be identified, including the integrity of solder bump bonding, degradation of polymer bumps, and high temperature processing requirement for the diffusion bonding. Other processes have also been demonstrated in the direct integration of high-aspect-ratio structures with microelectronics [8], [9]. In addition, efforts can be found in making movable LIGA structures by using an aligned molding and electroplating process [10] and a sacrificial layer etching process [11], [12]. These recent and on-going efforts indicate the importance of integrated electro-mechanical manufacturing.

A new batch transfer process based on flip-chip, electroplating and bonding is reported in this work. The advantages of this process include low-temperature processing, reliable bonding by batch manufacturing, and capability of making free-standing microstructures. The electroplating process takes place at a low temperature of $50^{\circ} \mathrm{C}$ such that possible damages due to high temperature processing are avoided. Flip-chip bonding via electroplating also shows good characteristics in strong bonding and good reliability. Furthermore, fixed microstructures can be transferred as movable ones such that device functionality is dramatically increased. As such, this widely applicable process is suitable for the integration of mechanical microstructures fabricated by LIGA or other processes with microelectronics massively and in parallel.

\section{The Flip-ChiP, Selective Bonding PROCESS}

A schematic diagram of the flip-chip, batch-assembly process is shown in Fig. 1. Two substrates, one has LIGA microstructures and the other has microelectronics, are to be assembled. In this demonstration example, a Pyrex glass is used as the IC substrate and the LIGA MUMP's service provided by MCNC [13] is used to fabricate the LIGA substrate. Shown in Fig. 1(a) is the glass substrate that is coated and patterned with $500 \AA$ chromium and $2000 \AA$ gold as the bonding pads by using mask $\# 1$. Chemical wet etching processes of $\mathrm{Au}$ and $\mathrm{Cr}$ are applied to etch the unwanted regions, respectively.

A $2.7-\mu \mathrm{m}$ thick sacrificial positive photoresist, AZ 1827 [14], that serves as the electrical isolation layer between circuitry and electroplating seed layer is coated and patterned by mask \#2. An electroplating seed layer consisting of 500- $\AA$ chromium and $2000-\AA$ gold is then deposited and patterned by mask \#3. In order to achieve good adhesion between the seed layer and the 


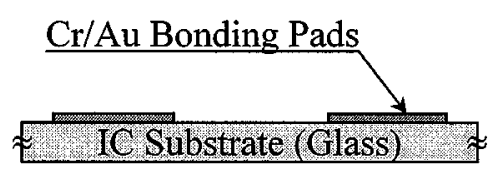

(a)

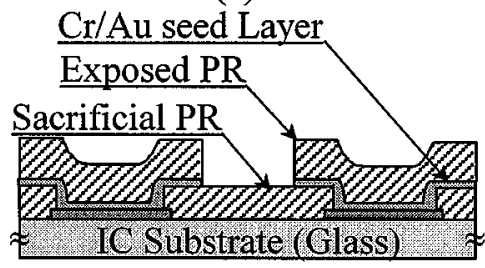

(b)

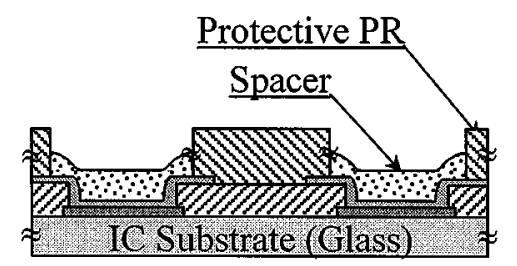

(c)

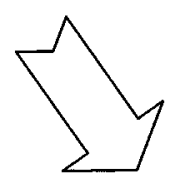

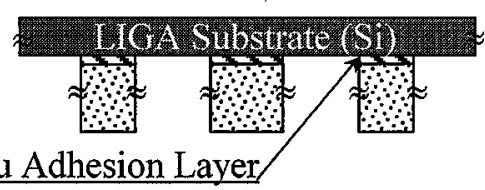

(d)

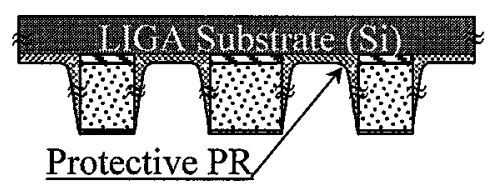

(e)

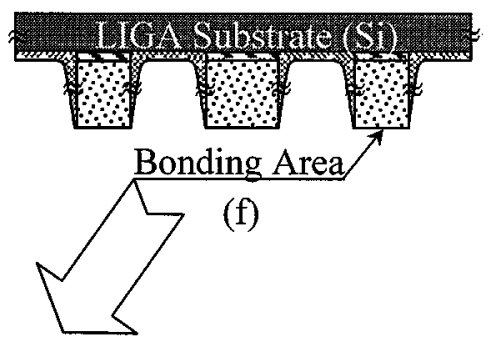

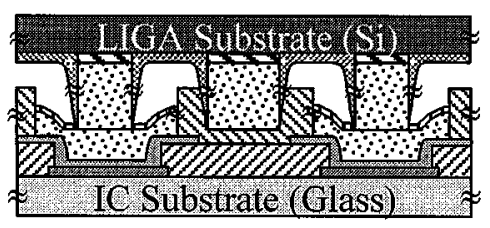

(g)

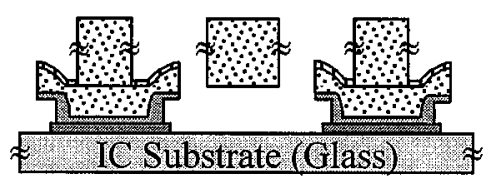

(h)

Fig. 1. The fabrication sequence.

bonding pads, oxygen plasma with excitation power of $100 \mathrm{~W}$ is applied for 60 seconds before the $\mathrm{Cr} / \mathrm{Au}$ evaporation process. Fig. 1(b) applies after these processes. In both lithography processes (mask \#2 and \#3), only one kind of positive photoresist, AZ 1827, is used. Therefore, a special step is introduced to keep the sacrificial photoresist from being removed. This is accomplished by introducing a blank exposure step right after the lithography steps by using mask \#3. As shown in Fig. 1(b), photoresist that is used to define the electroplating seed layer is exposed by the UV light and can be removed by the photoresist development process. On the other hand, sacrificial photoresist that is under the $\mathrm{Cr} / \mathrm{Au}$ seed layer during the blank exposure process is protected. After the wet etching processes to define the $\mathrm{Cr} / \mathrm{Au}$ seed layer, an added photoresist development process is used to remove the exposed photoresist. This way, the sacrificial photoresist remains on the substrate and the exposed photoresist is removed.

After removing the exposed photoresist, a $25-\mu \mathrm{m}$ thick, protective positive photoresist layer made of AZ 9620 is then coated and defined by using mask \#4. This layer is used to prevent unwanted deposition during the electroplating step. An oxygen plasma ashing process is applied afterwards to clean the bonding pad areas. A layer of 5- $\mu \mathrm{m}$ thick nickel is then selectively deposited by electroplating as the spacers (suspension gaps) as illustrated in Fig. 1(c). The thickness of the spacer layer determines the suspension gap between the movable LIGA microstructures and the IC substrate.

The second substrate uses a LIGA chip from the foundry service and it has a size of $10 \times 8 \mathrm{~mm}$. The flip-chip, cross sectional diagram is shown in Fig. 1(d). Photoresist is coated to protect the sidewall and top surface of LIGA microstructures. In order to obtain better step coverage, photoresist with lower viscosity is preferred. A positive photoresist, AZ 1805 is chosen and poured on the LIGA substrate. Two mechanisms, one is natural and the other is artificial, help the photoresist coating process. Capillary force, that is natural, drives the photoresist to fill small gaps. It is found that a waiting period of $5 \mathrm{~min}$. is required to assure the uniform distribution of photoresist by capillary force. 


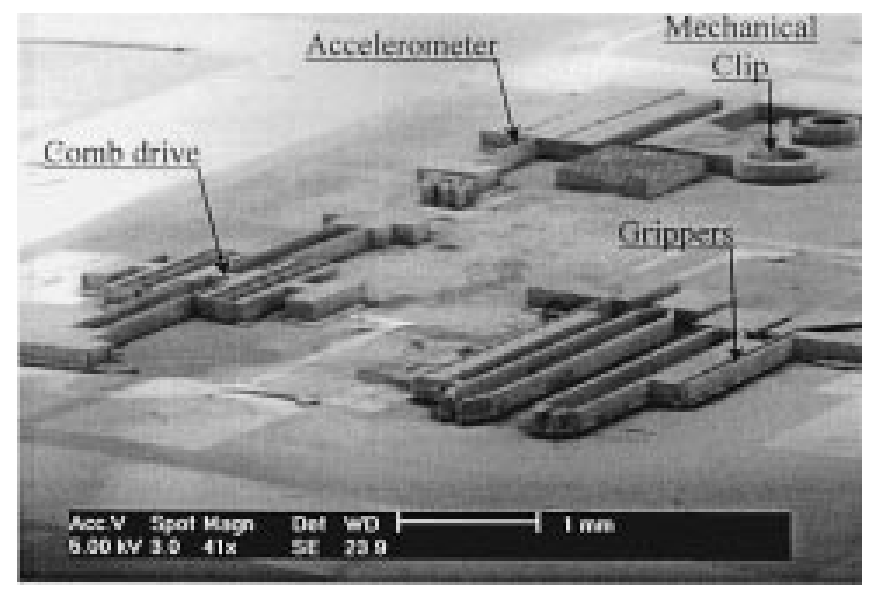

Fig. 2. Overview of flip-chip bonded LIGA microstructures on a glass substrate.

A slow spinning process, that is artificial, at $2000 \mathrm{rpm}$ for 30 seconds helps removing excessive photoresist for better uniformity. Fig. 1(e) applies after this step. Because of these high-aspect-ratio structures, photoresist is unevenly distributed on the sidewalls of LIGA microstructures.

In the flip-chip, selective bonding process, bonding areas on top of LIGA microstructures should be opened for bonding. Instead of going through lithography processes to open the unevenly distributed photoresist, a mechanical grinding process is applied by a regular planar grinder. A 1200 grid sand paper is chosen with the grinder running at $30 \mathrm{rpm}$. The sample is held by hand to provide the contact pressure and water is used as the lubricating fluid. The grinding process takes about five minutes until the top photoresist is removed as observed under an optical microscope. The sample is then rinsed by deionized water to clean the grinding debris. This step opens the top surface of LIGA microstructures for flip-chip bonding as shown in Fig. 1(f).

Both substrates are now aligned under an optical microscope manually, and fixed by a clip. In order to keep the two substrates from moving after the alignment process, a small amount of deionized water is applied at the contact interface to provide the adhesion force. The assembled substrates are put into nickel plating bath consisting of aqueous nickel sulfamate solution (1600 $\mathrm{ml})$, boric acid (24 g), and wetting agent (16 ml) [15]. The protective photoresist layer as illustrated in Fig. 1(g) is used to prevent unwanted electroplating by pressing the LIGA structure into the protective photoresist layer. Electroplating process at $50{ }^{\circ} \mathrm{C}$ with current density at $68.3 \mathrm{Am} / \mathrm{m}^{2}$ is conducted for 80 minutes to complete the bonding. The electroplated nickel fills the gap between two substrates and bonds them together. Acetone is used after the bonding process with an ultrasonic stripper to remove both sacrificial and protective photoresist. The copper adhesion layer that holds the LIGA microstructure with the original LIGA substrate is now etched away by using copper etchant which consists of $\mathrm{Cu} \mathrm{So}_{4}, \mathrm{NH}_{4} \mathrm{OH}$ and water. The whole process is completed as shown in Fig. 1(h) after these steps. The total number of masks for this batch transfer process is four and the estimated overall alignment tolerance is $20 \mu \mathrm{m}$ with manual operation.

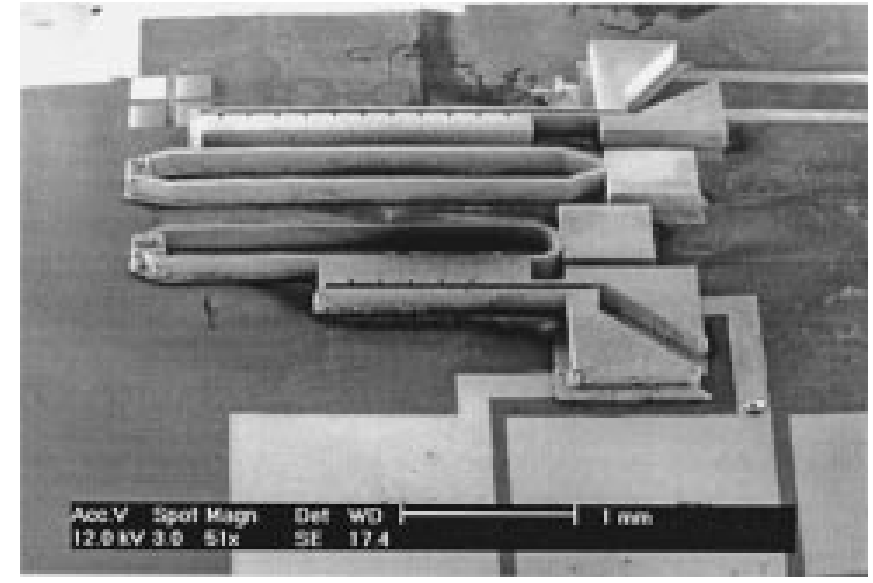

Fig. 3. The close view micrograph of two LIGA microgrippers and bonding pads.

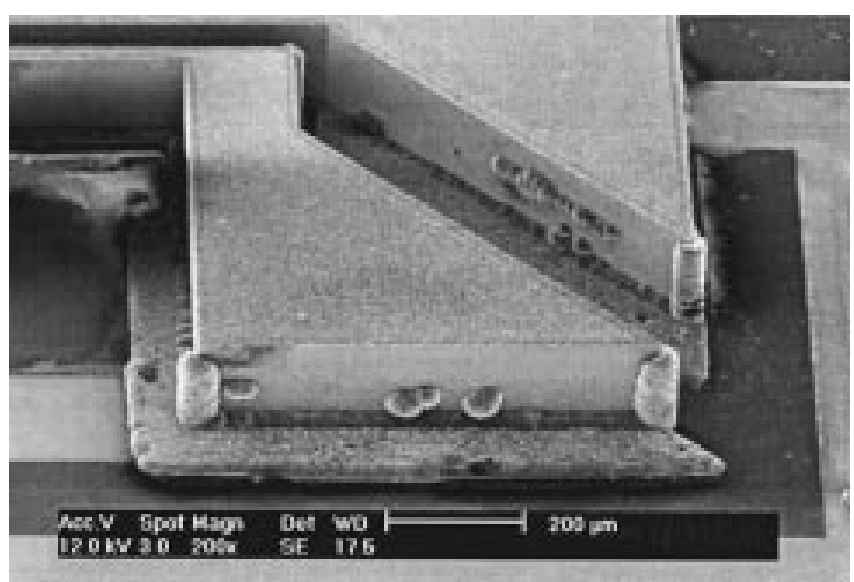

Fig. 4. The close view SEM micrograph showing the bonding interface and excessive electroplating.

Fig. 2 shows the SEM microphoto of several assembled LIGA microstructures, including two microgrippers, one comb-drive resonator, one accelerometer and one mechanical clip. The total transferable device area is about $10 \times 8 \mathrm{~mm}$ as limited by the LIGA foundry service. However, whole wafer transfer is feasible based on the demonstrated process. Fig. 3 shows two microgrippers, which are suspended $5 \mu \mathrm{m}$ above the substrate as predefined by the nickel spacer. These LIGA microgrippers have arm length of $2700 \mu \mathrm{m}$, width of $40 \mu \mathrm{m}$ and thickness of $200 \mu \mathrm{m}$. They are designed for actuation by means of electrothermal expansion [16]. Because of the selective bonding process, the anchors are fixed on the substrate but the microstructures are suspended and free to move. A close view SEM microphoto in Fig. 4 reveals the bonding interface, where the newly electroplated nickel fills the gap between LIGA microstructures and the IC substrate. Due to process imperfections such as cracks and damages on protective photoresist, excessive electroplating sometimes occurs as irregular beads on the sidewalls of microstructures as shown in Fig. 4. It is found that when the separation gap between two structures is smaller than $20 \mu \mathrm{m}$, this excessive deposition may connect the gap and cause electrical short circuit. 


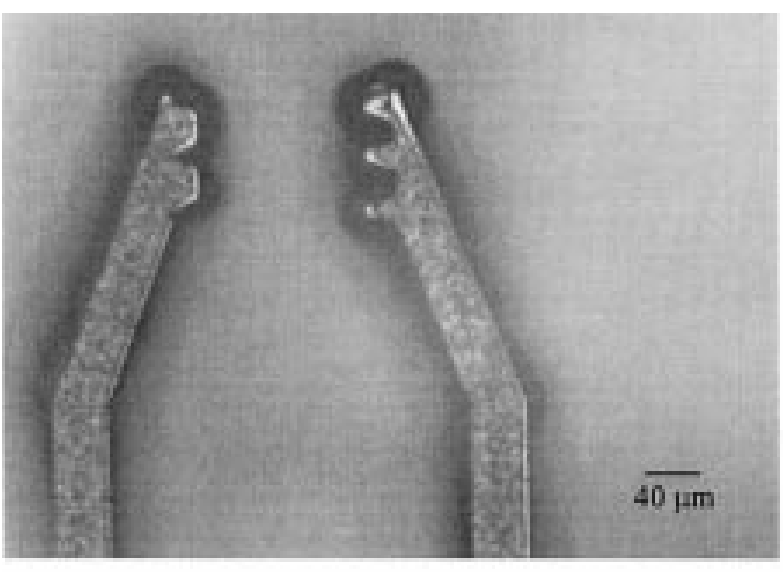

(a)

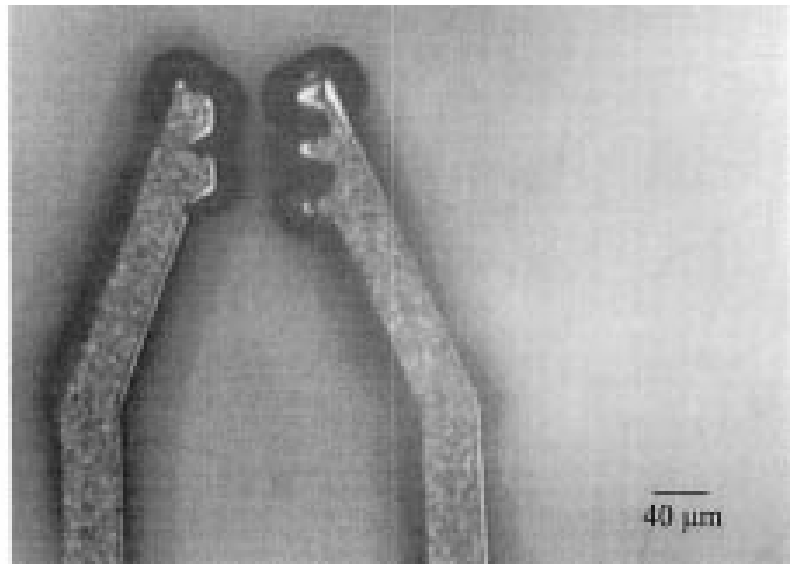

(b)

Fig. 5. (a) The tips of the LIGA microgripper before applying input power.(b) The tips of the LIGA micrographer after applying a 1.6-A input current.

\section{EXPERIMENTAL AND SimUlation REsUlts}

In this demonstration process, microgrippers are found to be functional. Both microaccelerometer and comb-drive resonator are not operational due to the excessive electroplating that shorted the small gaps of these microstructures. These microgrippers are high-aspect-ratio such that they are expected to deliver large force when compared with thermal actuators fabricated by surface-micromachining processes [16]. However, the driving current is extremely high for these microgrippers because of their low electrical resistance. Shown in Fig. 5(a) is the tip region of the LIGA microgripper before any electrical current is applied. Under a high input current, one microgripper arm moves to close the gap as shown in Fig. 5(b). These metal-based electrothermal actuators may not be desirable due to the low resistivity of metal and high current requirement for actuation. For example, based on the macro scale resistivity of nickel at $6.84 \mu \Omega \cdot \mathrm{cm}$, the LIGA microgripper has the resistance of only $0.03 \Omega$. Experimentally, the contact resistance between the electrical probe and the contact pad can be as high as $1.1 \Omega$. Therefore, when an input current is applied, the two contact pads become the major sources of heat generation.

Finite element analysis (FEA) is introduced to investigate the temperature distribution and displacement of LIGA microgripper under various input current levels. The analysis is performed on ANSYS 5.5 [17] with 2-D electrical-thermal elements. The bottom of the glass substrate is set at room temperature and both heat conduction and heat convection are considered as the heat dissipation mechanisms. In the numerical simulation, extra heat generated by contact resistance is modeled by putting two additional heat generation sources at the anchors of the LIGA microgripper. The temperature distribution as predicted in Fig. 6 shows a highest temperature of $1211^{\circ} \mathrm{C}$ at the anchor of the thinner beam when the input current is $1.6 \mathrm{~A}$. This is close to the melting temperature of nickel at $1455^{\circ} \mathrm{C}$. Under the same input current, the tip of the microgripper has a temperature of $286^{\circ} \mathrm{C}$. The operation of the LIGA microgripper clearly depends on various material properties with respect to temperature variations, including electrical resistivity, Young's modulus, and thermal expansion coefficient of nickel. Due to the lack of material database in the micro scale, the numerical char- acterization of these LIGA microgrippers is only appropriate as first-order estimation.

In order to characterize the microgripper performance, a current versus displacement (at the tip of the microgripper) and FEA results are plotted in Fig. 7. When the input current is at $1.6 \mathrm{~A}$, the movement of the microgripper is $92 \mu \mathrm{m}$ and the device failed to operate. The measured power consumption is $3.93 \mathrm{~W}$ at this point. According to FEA simulation, the anchor at the thinner beam reaches $1211^{\circ} \mathrm{C}$. This high temperature may trigger structural failure due to thermal mismatch between nickel and glass as well as the possible softening of glass. After the experiment, cracks are found around the anchors as shown in Fig. 8. Moreover, the anchor is found to be detached from the glass substrate as the device failure mechanism.

\section{DISCUSSIONS}

During the experiments, it is observed that for any gap less than $20 \mu \mathrm{m}$, excessive electroplating may occur and short the nickel structure. This gives a good guideline for various design considerations. For example, alignment between LIGA and IC substrates is required during the flip-chip bonding process. In this work, the alignment process is performed manually under an optical microscope and the estimated alignment error is $20 \mu \mathrm{m}$. Therefore, to prevent short circuitry due to excessive electroplating, the minimum distance between two bonding pads on the IC substrate should be at least $40 \mu \mathrm{m}$ for accounting both the possible alignment error of $20 \mu \mathrm{m}$ and excessive deposition of $20 \mu \mathrm{m}$. Moreover, in the structure design, any gap of adjacent microstructures should be at least $20 \mu \mathrm{m}$ to prevent possible electrical short circuit due to the excessive deposition. It should be pointed out that a glass substrate is used in this work such that manual alignment is possible under an optical microscope. If a double-side aligner or advanced flip-chip bonding equipment is used for the alignment step, the alignment error is expected to be further reduced.

To eliminate the problem of excessive deposition, protective photoresist should be coated on the LIGA microstructure as shown in Fig. 1(e). After this coating process, a planar grinding process is used to open the top surface for bonding. One fabrication result after the mechanical grinding process is shown 


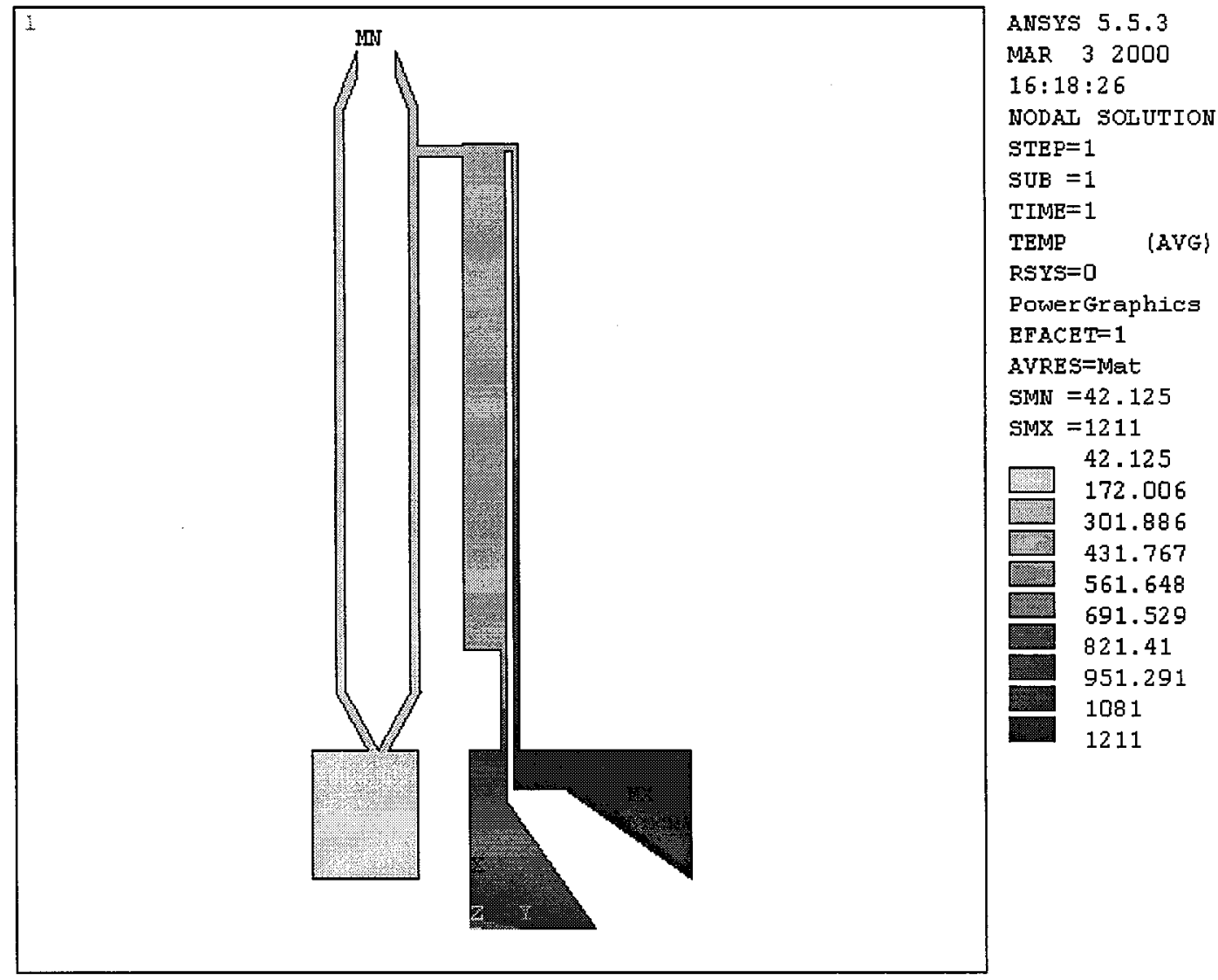

Fig. 6. FEA simulation result of temperature distributions under an input current of $1.6 \mathrm{~A}$.

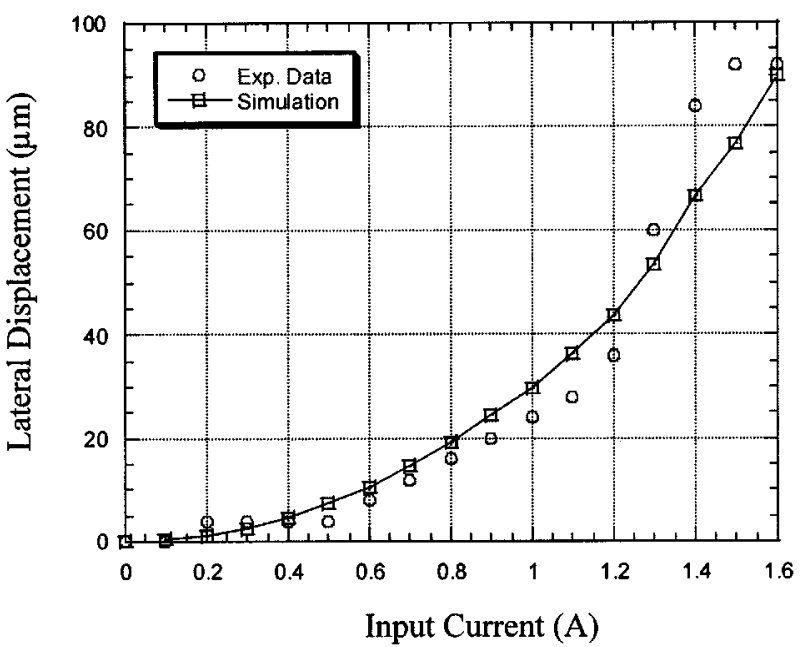

Fig. 7. Experimental and FEA simulation results of lateral displacement versus the input current for the thermal actuator.

in the SEM microphoto of Fig. 9. In this figure, a comb-drive resonator with finger width of $30 \mu \mathrm{m}$ and separation gap of $10 \mu \mathrm{m}$ between two comb structures is coated with photoresist. It is observed that photoresist is accumulated between the small separation gaps and the coating is relatively uniform at other areas. A closed view SEM microphoto is shown in Fig. 10. It reveals that a strip of photoresist about $20-\mu \mathrm{m}$ wide measured from the top surface of LIGA microstructure is peeled off probably during the grinding process. This process defect is later compensated by covering the LIGA microstructure with the $25-\mu \mathrm{m}$ thick protective photoresist in the flip-chip bonding step as illustrated in Fig. 1(g). However, there are still two common places that may have excessive deposition. First, corners of LIGA microstructures generate high electric field during the electroplating process such that it is easy to introduce excessive electroplating at corners. Second, the top surface areas that are not designed as selective bonding pads but were opened during the grinding process are possible electroplating sites. Although a layer of protective photoresist is utilized to allow the immersion and protection of these areas during the bonding process, excessive electroplating may still occur due to process and photoresist imperfections. To achieve excellent protection of the microstructure and to open only the bonding areas during the electroplating process require further process development.

In order to investigate the bonding strength and bonding interface, various microstructures are forcefully detached from the glass substrate by using a probe from the probe station. Three typical results are identified as illustrated in Fig. 11. First, the $\mathrm{Cr} / \mathrm{Au}$ bonding pad on the glass substrate is removed as shown on the site of the mechanical clip in Fig. 11. This indicates that the bonding strength via electroplating maybe stronger than the adhesion force between $\mathrm{Cr} / \mathrm{Au}$ and glass substrate. Second, the probe cannot break the bond and remove the microstructure as exemplified by the fixed, upper electrode of the micro ac- 


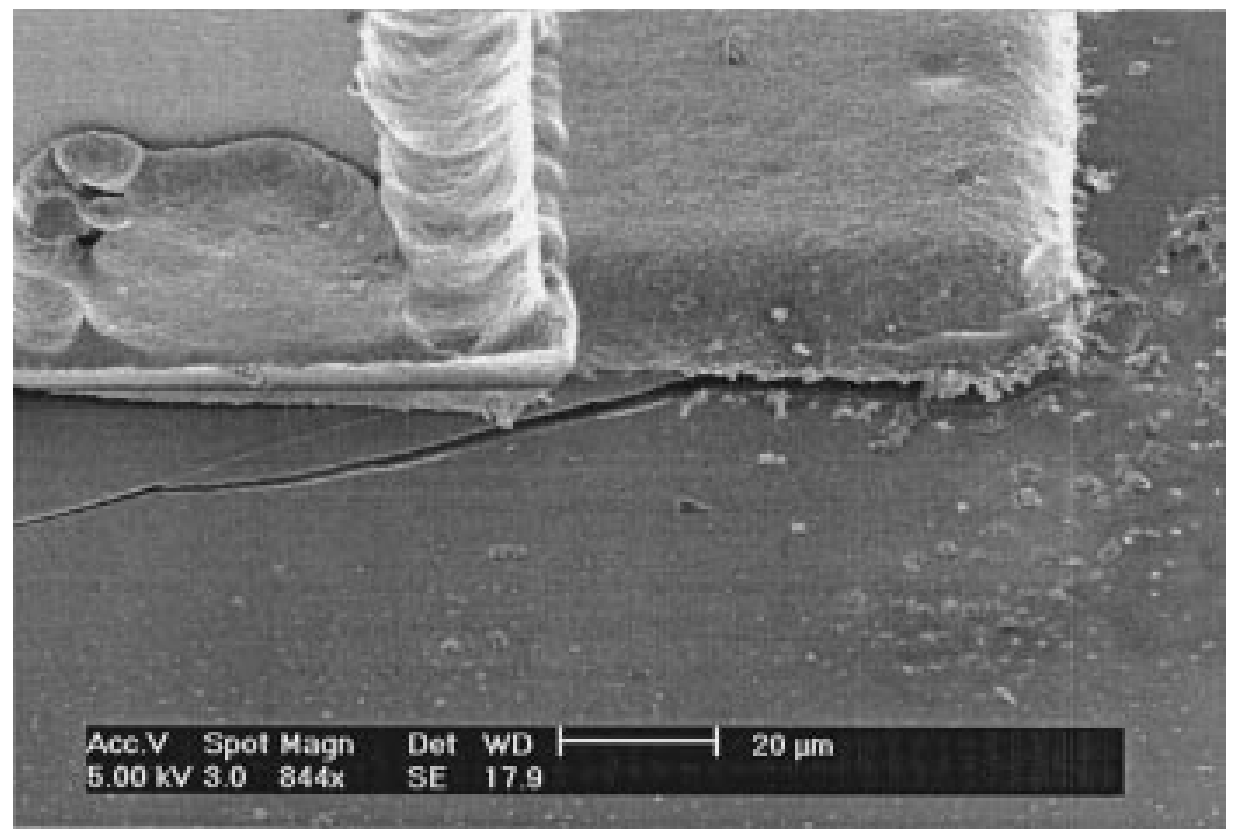

Fig. 8. Cracks are found on the glass substrate after high temperature operations.

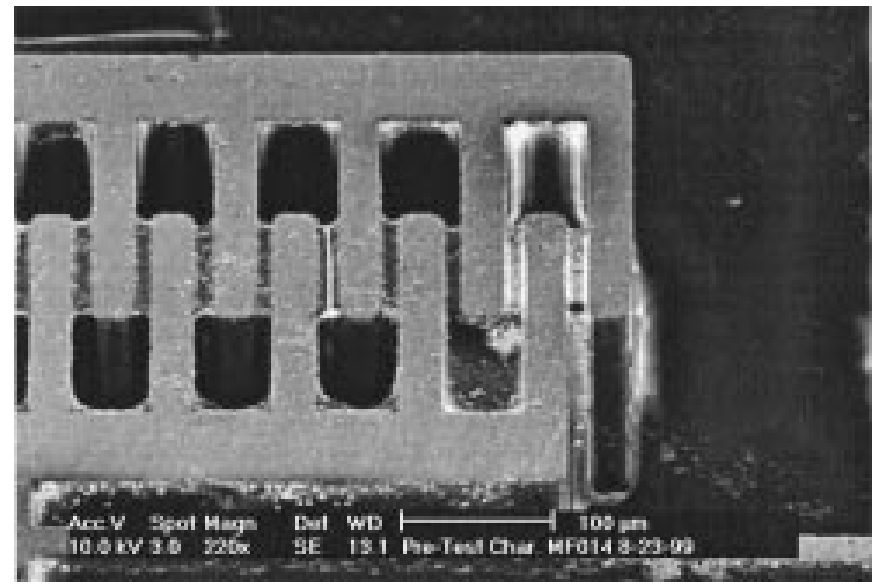

Fig. 9. The SEM microphoto of the LIGA microstructure after the protective photoresist coating and mechanical grinding process.

celerometer as shown in Fig. 11. Even after several trials, the microstructure remains intact and cannot be detached. This implies structural defects are too small to cause cracks to propagate under external force. Third, the microstructure is detached from the electroplating interface and the spacer layer and the $\mathrm{Cr} / \mathrm{Au}$ bonding pads stay on the glass substrate. This condition is shown in Fig. 11 at the lower electrode site of the micro accelerometer. Although further studies are required to characterize these various bonding results, observations are made to provide qualitative explanations. In this flip-chip assembly process, electrolyte has to be able to diffuse into the bonding gaps for electroplating and bonding. However, low concentration of nickel ion is expected if the bonding area is far away from the electrolyte supply. Intuitively, devices at the global center of the LIGA substrate and areas at the central region of a bonding pad may have this problem of poor nickel ion supply. As a re- sult, the bonding quality is affected. In order to improve the diffusion process, pulse electroplating [18] has been suggested to provide ample diffusion time for nickel ion to reach the bonding area. Previously, an electroplating experiment has been set up to characterize the effective bonding distance [19]. It is found that the characteristic bonding length for reliable bonding is $200 \mu \mathrm{m}$ into the bonding pad edges. Therefore, bonding pads used in this paper are designed by using this characteristic bonding length and complete bonding is expected. Fig. 11 also provides clear evidence of excessive electroplating between small gaps of the micro accelerometer. A close view SEM microphoto at the far end edge in Fig. 12 reveals that excessive electroplating not only occurs in the small gap of the moving mass but also shorts the fixed electrodes and the moving mass. Furthermore, excessive electroplating can also be identified at the bottom edges of the moving mass. This may prevent the mass from moving due to high frictional force.

Nickel electroplating is the combination/competition of two processes, the growth along the epitaxial orientation of the plating surface and the nickel crystallites of various crystal orientations [20]-[22]. The face of preferential growth is determined by the electrode potential and $\mathrm{pH}$ value of the electrolyte. Froment et al. [23] reported that five different textures may be formed as affected by the $\mathrm{pH}$ value of the plating bath and the current density during electroplating. These parameters clearly affect the electroplating and bonding process and should be studied further. The typical growth pattern of nickel in this selective bonding via electroplating process can be shown in Fig. 13. In this corner region, electric field is expected to be strong such that large-size of nickel grains are observed. The typical grain size is about $10 \mu \mathrm{m}$ at the corner regions as compared with $1 \mu \mathrm{m}$ of grain size at the bonding interface under the microstructures. Although the growth of nickel grain is not the main focus of this work, investigations should be continued 


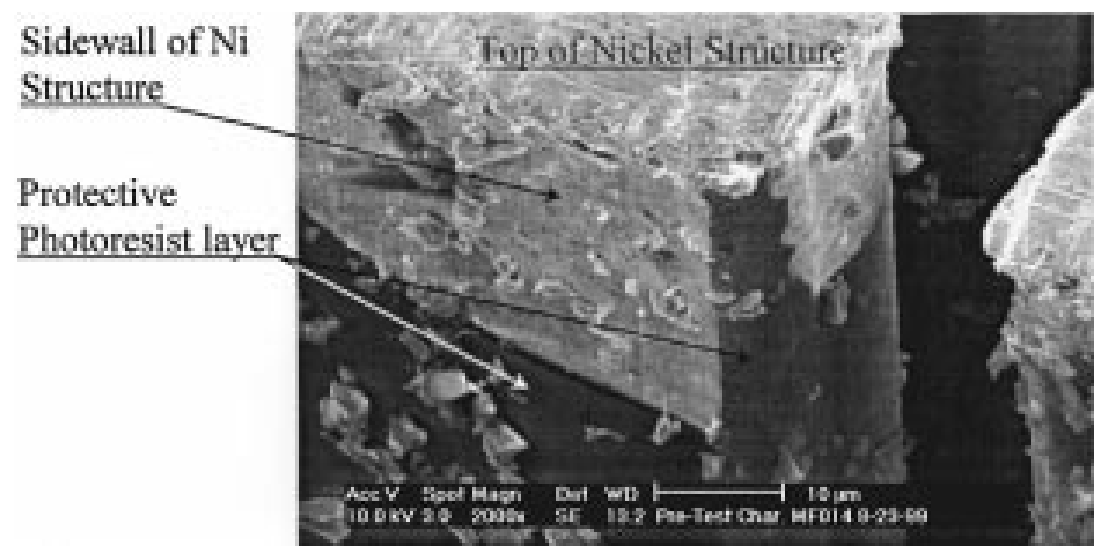

Fig. 10. The close view SEM microphoto showing that part of the protective photoresist layer was peeled-off after the grinding process.

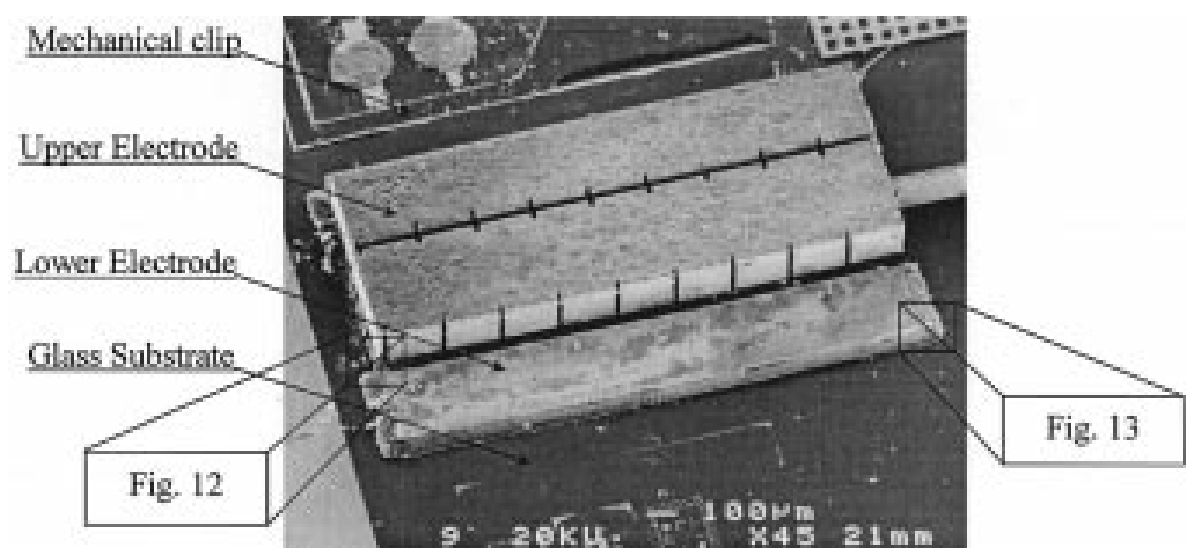

Fig. 11. The SEM microphoto showing detached bonding interfaces on the glass substrate.

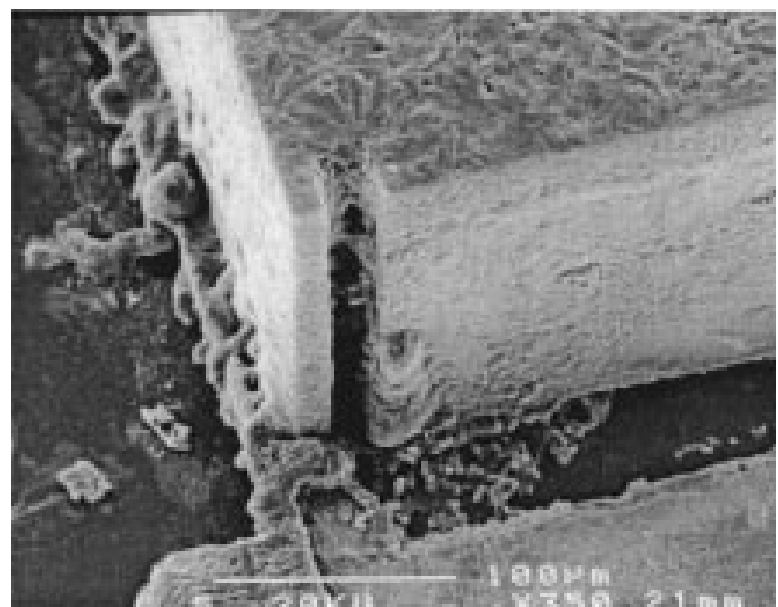

Fig. 12. Excessive electroplating is found at various places.

in the electrolyte concentration, process temperature, time and the growth of nickel with respect to the bonding characteristics.

\section{CONCLUSIONS}

LIGA microstructures have been successfully transferred to a glass substrate by means of selective bonding via electroplating. A selective nickel bonding process is developed as the demonstration example in this work with processing temperature at 50 ${ }^{\circ} \mathrm{C}$ and current density at $68.3 \mathrm{Am}^{2}$. Experimental results show

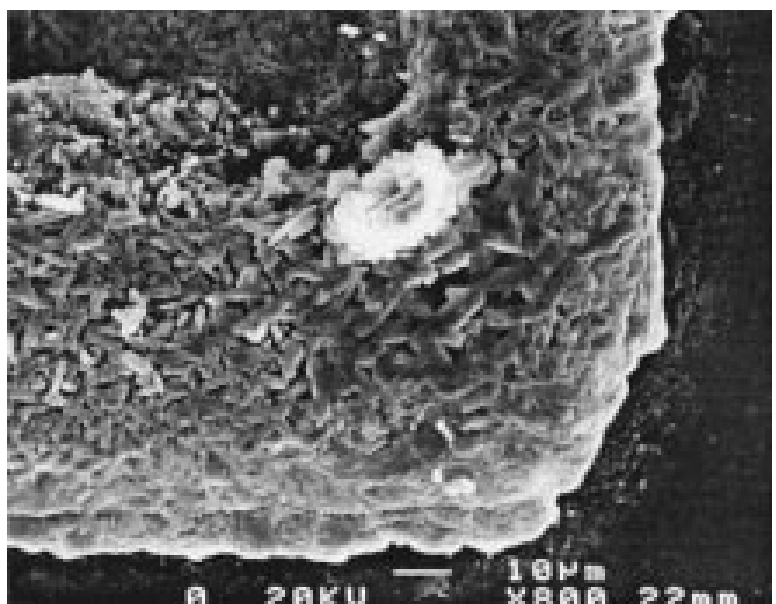

Fig. 13. Large nickel grains are found at the corner regions.

that LIGA microgrippers are operative after the batch transfer process and a maximum displacement of $92 \mu \mathrm{m}$ is achieved under an input current of 1.6 A. An FEA simulation program is established to model the microgripper. It is found that the highest temperature is about $1211^{\circ} \mathrm{C}$ at the anchor of the thinner beam when the microgripper moves $92 \mu \mathrm{m}$. Process iterations are necessary to improve the yield for successful transfer and to establish the design rules such as the minimum distance between two microstructures to avoid excessive deposition as observed in this work. In summary, this flip-chip assembly process enables 
a new class of integrated electro-mechanical manufacturing that have potential applications for both MEMS and mesoscopic systems.

\section{ACKNOWLEDGMENT}

The authors would like to thank R. Wilson for taking SEM pictures.

\section{REFERENCES}

[1] T. A. Core, W. K. Tsang, and S. Sherman, "Fabrication Technology for an Integrated Surface-Micromachined Sensor," Solid State Technol., pp. $39-47,1993$.

[2] W. Yun, R. T. Howe, and P. R. Gray, "Surface Micromachined, Digitally Force-balanced Accelerometer with Integrated CMOS Detection Circuitry," in Proc. 5th IEEE Solid-State Sensor and Actuator Workshop, 1992, pp. 126-131.

[3] K. A. Shaw and N. C. MacDonald, "Integrating SCREAM Micromachined Devices with Inregraed Circuits," in 1996 Micro Electro Mechanical Systems Workshop, pp. 44-48.

[4] J. Smith, S. Montague, J. Sniegowski, R. Manginell, P. McWhorter, and R. Huber, "Characterization of the embedded micromechanical device approach to the monolithic integration of mems with cmos," SPIE, vol. 2879, 1996.

[5] A. Singh, D. A. Horsley, M. B. Cohn, A. P. Pisano, and R. T. Howe, "Batch Transfer of Microstructures using Flip-Chip Solder Bump Bonding," in Proc. 1997 Int. Conf. Solid-State Sensors and Actuators. pp. 265-268.

[6] K. W. Oh and C. H. Ahn, "Development of An Innovative Flip-Chip Bonding Technique Using Micromachined Conductive Polymer Bumps," in Proc. Solid-State Sensor and Actuator Workshop, Hilton Head Island, SC, 1998, pp. 170-173.

[7] T. R. Christenson and D. T. Schmale, "A Batch Wafer Scale LIGA Assembly and Packaging Technique via Diffusion Bonding," in Proc. IEEE Int. Conf. MEMS, 1999, pp. 476-481.

[8] H. H. Langen, T. Masuzawa, and M. Fujino, "Self-Aligned Machining and Assembly of High Aspect Ratio Microparts into Silicon," in 1995 Micro Electro Mechanical Systems Workshop, 1995, pp. 250-255.

[9] A. Both, W. Bacher, and M. Heckele et al., "Molding Process with High Alignment Precision for the LIGA Technology," in 1995 Micro Electro Mechanical Systems Workshop, 1995, pp. 186-190.

[10] O. Roetting, M. Heckele, and W. Bacher, "Technology for movable microstructures - Transfer of laboratory process to industrial production," in Microsystem Technologies. New York: Springer-Verlag, 1998, vol. 4, pp. $120-1$.

[11] S. Takimoto, R. Kondo, K. Suzuki, and S. Sugiyama, "Fabrication of Micromotors Using LIGA process," in Proc. Int. Symp. Micromechatronics Human Science, Nagoya, Japan, 1999, pp. 221-6.

[12] W. Qu, C. Wenzel, and G. Gerlach, "Fabrication of a 3D differentialcapacitive acceleration sensor by UV-LIGA," Sens. Actuators A. Phys., vol. A77, no. 1, pp. 14-20, 1999.

[13] Multi-User MEMS Processes (MUMPS) Introduction and Design Rules, rev. 3, MCNC MEMS Technology Applications Center Research, Triangle Park, NC, Oct. 1994.
[14] AZ Electronic Materials, Business Unit Electronic Materials, Somerville, NJ, USA

[15] MacDermid, Inc, , Waterbury, CT.

[16] J. H. Comtois, M. A. Michalicek, and C. C. Barron, "Electrothermal actuators fabricated in four-level planarized surface micromachined polycrystalline silicon," Sens. Actuators A, Phys., vol. A70, no. 1-2, pp. 23-31, 1998

[17] , ANSYS,Inc, Canonsburg, PA.

[18] J.-Cl. Puippe and F. Leaman, Theory and Practice of Pulse Plating: American Electroplaters and Surface Furnishers Soc, 1986.

[19] L. Pan, L. Lin, and J. Ni, "A Flip-Chip LIGA Assembly Technique via Electroplating," in Book of Abstract of HARMST '99, 1999, pp. 108-109.

[20] U. Klement, U. Erb, and K. T. Aust, "Investigations of the Grain Growth Behavior of Nanocrystalline Nickel," NanoStructured Materials, vol. 6, pp. 581-584, 1995.

[21] R. Weil and J. B. C. Wu, "Structures of Thin Nickel Electrodeposits Epitaxially Grown on Cube-Textured Copper Substrates," Plating (USA), vol. 60, no. 6, pp. 622-626, 1973.

[22] E. Budevski, G. Staikov, and W. J. Lorenz, Electrochemical Phase Formation and Growth: An Introduction to the Initial Stages of Metal Deposition. New York: VCH Publishers, 1996.

[23] J. Amblard, I. Epelboin, M. Froment, and G. Maurin, J. Appl. Electrochem., vol. 9, p. 233, 1979.

Li-Wei Pan was born in Taiwan, R.O.C. He received the B.S. degree in mechanical engineering from the National Chiao-Tung University, Hsinchu, Taiwan, R.O.C., in 1991, and the M.S. degree in mechanical engineering and applied mechanics from The University of Michigan, Ann Arbor, in 1996 . He is currently working toward the Ph.D. degree in the mechanical engineering at the University of Michigan.

His research interests include electroplating bonding, hybrid MEMS system, IC and MEMS integration, LIGA microsensors and microactuators. His current research focuses on the integration of high-aspect microstructures.

Liwei Lin received the M.S. and Ph.D. degrees in mechanical engineering from the University of California, Berkeley, in 1991 and 1993 respectively.

He worked on the research and development of microsensors at BEI Electronics Inc. USA from 1993 to 1994. From 1994 to 1996, he was an Associate Professor at the Institute of Applied Mechanics, National Taiwan University, Taiwan. From 1996 to 1999, he was an Assistant Professor at the Mechanical Engineering and Applied Mechanics Department at the University of Michigan, Anne Arbor. Since 1999, he has been an Assistant Professor at the Mechanical Engineering Department, and Co-Director at the Berkeley Sensor and Actuator Center, NSF/Industry/University Research Cooperative Center, at the University of California, Berkeley. His research interests are in microelectromechanical systems, including design, modeling and fabrication of microstructures, microsensors and microactuators.

Dr. Lin is the recipient of the 1998 NSF CAREER Award for research in MEMS packaging and the 2000 ASME Journal of Heat Transfer Best Paper Award for his work on micro scale bubble formation. He led the effort in establishing the MEMS sub-division in ASME and is currently serving as the Vice-Chairman of the Executive Committee for the MEMS sub-division. He holds six U.S. patents in the area of MEMS 